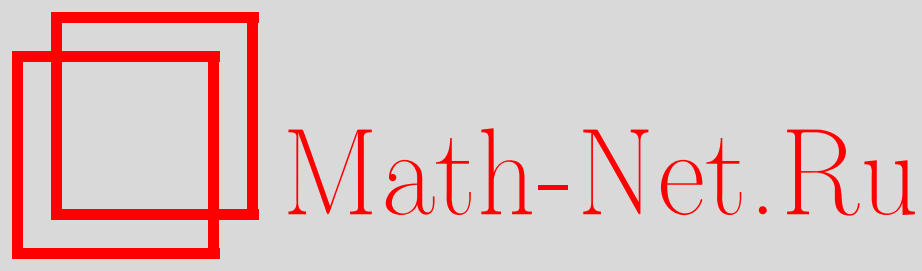

М. Г. Иванов, Двоичное представление координаты и импульса в квантовой механике, ТМФ, 2018, том 196, номер 1, 70-87

DOI: https://doi.org/10.4213/tmf9444

Использование Общероссийского математического портала Math-Net.Ru подразумевает, что вы прочитали и согласны с пользовательским соглашением http://www.mathnet.ru/rus/agreement

Параметры загрузки:

IP : 52.6 .47 .48

26 апреля 2023 г., 16:22:22

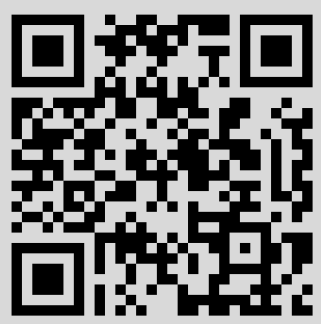




\title{
ДВОИЧНОЕ ПРЕДСТАВЛЕНИЕ КООРДИНАТЫ И ИМПУЛЬСА В КВАНТОВОЙ МЕХАНИКЕ
}

\begin{abstract}
Для моделирования квантовой системы с непрерывными степенями свободы на квантовом компьютере, основанном на кубитах, необходимо свести непрерывные наблюдаемые (в первую очередь координаты и импульсы) к двоичным наблюдаемым. Данная задача рассматривается на основе разложения квантовых наблюдаемых в ряд по степеням двойки, аналогичный двоичному представлению вещественных чисел. Коэффициенты ряда ("цифры") при этом являются ортогональными проекторами. Исследуются соответствующие квантовомеханические операторы и соотношения между ними. Показано, что двоичное разложение квантовых наблюдаемых автоматически приводит к перенормировке некоторых расходящихся интегралов и рядов (приписыванию им конечных значений).
\end{abstract}

Ключевые слова: квантовые вычисления, кубит, двоичное разложение, перенормировка.

DOI: https://doi.org/10.4213/tmf9444

\section{1. ВВЕДЕНИЕ}

При обсуждении будущих применений квантовых компьютеров зачастую внимание сосредотачивается преимущественно на задачах криптоанализа. Однако к моменту появления достаточно мощных квантовых компьютеров, вероятно, будет повсеместно внедрена постквантовая криптография, стойкая к криптоанализу на квантовом компьютере [1]. В связи с этим можно ожидать, что основное применение квантовых компьютеров окажется "мирным" (не связанным со взломом шифров), в частности, большое внимание в соответствии с исходной идеей Фейнмана [2] будет уделяться моделированию квантовых систем.

Исследование выполнено за счет гранта Российского научного фонда (проект 16-11-00084) в Московском физико-техническом институте (государственном университете).

* Московский физико-технический институт (государственный университет), Долгопрудный, Московская обл., Россия. E-mail: ivanov.mg@mipt.ru 
Моделирование квантовых систем - очень актуальная задача с точки зрения практических применений, таких как квантовая химия, создание новых материалов, квантовая биофизика, разработка новых лекарств, ядерная физика и физика элементарных частиц. Многие из этих приложений требуют дискретизации непрерывных квантовых наблюдаемых, координат и импульсов, которые в квантовом компьютере должны описываться с помощью совокупности дискретных квантовых ячеек (кубитов и/или кутритов и других кудитов). Конечно, операторы импульса являются дифференциальными операторами, для их дискретизации можно использовать хорошо известные из вычислительной математики разностные схемы (см., например, работы [3], [4]). Однако такой прямолинейный подход не учитывает специфики квантовой механики, в которой оператор импульса является генератором сдвигов по соответствующей координате.

В настоящей статье строится представление непрерывных квантовых наблюдаемых на основе разложения в ряд по степеням двойки, аналогичного двоичному представлению вещественных чисел. Отдельная двоичная цифра разложения наблюдаемой при этом сама является наблюдаемой и описывается одним квантовым битом. Операторы двоичных цифр координаты и импульса на решетке и на прямой построены в явном виде, для них получены коммутационные соотношения. Построено двоичное интегральное представление вещественных чисел и квантовых наблюдаемых. При представлении наблюдаемых в виде двоичных рядов и интегралов некоторым формально расходящимся выражениям естественным образом приписываются конечные значения, т. е. вводится перенормировка. Такой способ перенормировки можно применить к конечным величинам (что связано с неоднозначностью выбора представителей пространства $\mathbb{Z}_{N}$ ), и это позволяет вычислять перенормированные величины на решетке.

В разделе 2 вводятся координаты и импульсы на конечной одномерной решетке размера $2^{n}$, причем оператор импульса является генератором сдвига по координате. В разделе 3 операторы координаты и импульса (как на решетке, так и на прямой) представляются в виде рядов по степеням двойки, коэффициенты разложения (операторы двоичных цифр) строятся явно. Для числа на прямой по аналогии с двоичным разложением строится интегральное представление. Исследуются возникающие при этом процедуры перенормировки (приписывания конечного значения рядам и интегралам). В разделе 4 исследуются коммутационные соотношения цифр координаты и импульса. Раздел 5 представляет собой заключение. В конце статьи помещены три приложения, которые содержат детали вычислений явного вида операторов цифр координаты и импульса, а также вычисления, с помощью которых можно проверить интегральное двоичное представление чисел на прямой, и примеры матриц цифр координаты и импульса для решеток размером $2^{1}, 2^{2}$ и $2^{3}$.

\section{2. КООРДИНАТЫ И ИМПУЛЬСЫ НА КОНЕЧНОЙ РЕШЕТКЕ}

Здесь и далее мы будем использовать координатное представление (если не оговорено иное) и полагать для постоянной Планка $h=1, \hbar=1 / 2 \pi, 1 / \hbar=2 \pi$.

2.1. Координатная решетка. Будем считать, что координата описывается с помощью $n$ цифр-кубитов, при этом решетка по координате состоит из $N=2^{n}$ узлов, которые мы считаем расположенными по кругу (после последнего идет первый). 
Положим, что шаг координатной решетки составляет $\Delta x=2^{-n_{-}}$, тогда период координатной решетки равен $\Xi=N \Delta x=2^{n_{+}}, n_{+}=n-n_{-}$. Считаем, что значения $x$ меняются от 0 до $\Delta x \cdot(N-1)$.

На координатной решетке вводится естественная операция сложения, наследуемая из $\mathbb{Z}_{N}$, для которой $x=x+\Xi$. Можно использовать и другие представления решетки $\Delta x \cdot \mathbb{Z}_{N}$ вещественными числами. Например, с учетом эквивалентности $x$ и $x+\Xi$ далее нам понадобится представление, при котором $x$ меняется от $-\Delta x \cdot N / 2$ до $+\Delta x \cdot(N / 2-1)$. Ряд для координаты на конечной решетке является конечным:

$$
x=\sum_{s=-n_{-}}^{n_{+}-1} x_{s} 2^{s}=\sum_{s=-n_{-}}^{n_{+}-1} c(s, x) 2^{s} .
$$

Здесь $x_{s}=c(s, x)$ - цифра с номером $s$ в двоичном разложении числа $x$. Иногда мы будем указывать диапазон степеней двойки, который задает решетку, и писать $x_{s}=c_{n_{-} n_{+}}(s, x)$.

Введем координатный базис $\{|x\rangle\}_{x \in \Delta x \cdot \mathbb{Z}_{N}}$ для функций, заданных на решетке:

$$
\hat{x}|x\rangle=x|x\rangle, \quad\left\langle x^{\prime} \mid x^{\prime \prime}\right\rangle=\delta_{x^{\prime}, x^{\prime \prime}}, \quad \psi(x)=\langle x \mid \psi\rangle, \quad x \in \Delta x \cdot \mathbb{Z}_{N} .
$$

Волновые функции (кет-векторы) будем представлять в виде столбцов, строки которых упорядочены в порядке убывания $x$. Так, если $x$ меняется от 0 до $(N-1) \Delta x$,

$$
\psi(x)=\left(\begin{array}{c}
\psi((N-1) \Delta x) \\
\psi((N-2) \Delta x) \\
\vdots \\
\psi(\Delta x) \\
\psi(0)
\end{array}\right) .
$$

2.2. Импульсная решетка. Оператор импульса $\hat{p}$ мы определим как генератор сдвига $\widehat{T}_{A}$ по координатной решетке:

$$
\widehat{T}_{A} \psi(x)=\psi(x+A), \quad \widehat{T}_{A}=e^{2 \pi i A \hat{p}}, \quad A \in \Delta x \cdot \mathbb{Z} .
$$

Такие операторы рассматривались еще в классической книге Вейля [5] и позже более подробно в статье Швингера [6].

В силу периодичности координатной решетки сдвиг на период $\Xi$ должен представлять собой тождественное преобразование, т. е. для собственных чисел оператора $\hat{p}$ имеем $\Xi \cdot p \in \mathbb{Z}$. Это дает нам шаг по импульсу $\Delta p$,

$$
\Xi \cdot \Delta p=1, \quad \Delta p=2^{-n_{+}}, \quad \Delta p \cdot \Delta x=\frac{1}{N}=2^{-n} .
$$

Количество точек в спектре импульса такое же, как для координаты, т. е. для импульса мы имеем периодическую решетку с тем же числом узлов, но с другим периодом $\Pi=\Delta p \cdot N=2^{n_{-}}, \Pi \Xi=N$. Импульсную решетку обозначим как $\Delta p \cdot \mathbb{Z}_{N}$. Ряд для импульса тоже является конечным:

$$
p=\sum_{r=-n_{+}}^{n_{-}-1} p_{r} 2^{r}=\sum_{r=-n_{+}}^{n_{-}-1} c(r, p) 2^{r} .
$$


Здесь $p_{r}=c(r, p)$ - цифра с номером $r$ в двоичном разложении числа $p$. Иногда мы будем указывать диапазон степеней двойки, который задает решетку, и писать $p_{r}=c_{n_{+} n_{-}}(r, p)$.

2.3. Минимальный сдвиг. Минимальный сдвиг $\widehat{T}_{\Delta x}-$ это сдвиг на шаг решетки $\Delta x$, любой другой сдвиг на данной решетке - это степень $\widehat{T}_{A}=\left(\widehat{T}_{\Delta x}\right)^{A / \Delta x}$, где $A / \Delta x \in \mathbb{Z}_{N}$ :

$$
\widehat{T}_{A} \psi(x)=\psi(x+A), \quad \widehat{T}_{A}|x\rangle=|x-A\rangle, \quad\left\langle x^{\prime}\left|\widehat{T}_{A}\right| x^{\prime \prime}\right\rangle=\delta_{x^{\prime}, x^{\prime \prime}-A}=\delta_{x^{\prime}+A, x^{\prime \prime}} .
$$

При этом

$$
\widehat{T}_{\Delta x} \psi(x)=\widehat{T}_{\Delta x}\left(\begin{array}{c}
\psi((N-1) \Delta x) \\
\psi((N-2) \Delta x) \\
\vdots \\
\psi(\Delta x) \\
\psi(0)
\end{array}\right)=\left(\begin{array}{c}
\psi(0) \\
\psi((N-1) \Delta x) \\
\psi((N-2) \Delta x) \\
\vdots \\
\psi(\Delta x)
\end{array}\right)=\psi(x+\Delta x) .
$$

Сумма $x+\Delta x$ считается в смысле $x \in \Delta x \cdot \mathbb{Z}_{N}$, т. е. это циклический сдвиг функции на решетке вниз на одну позицию.

Собственные числа оператора минимального сдвига суть корни степени $N$ из единицы, они связаны с собственными числами оператора импульса (который пока не вводился нами явно):

$$
\lambda^{N}=1, \quad \lambda_{p}=e^{2 \pi i \Delta x \cdot p}=e^{2 \pi i \Delta x \Delta p \cdot p / \Delta p}=\left(\lambda_{\Delta p}\right)^{p / \Delta p},
$$

где мы учли, что $\Delta x \Delta p=1 / N$ и $p / \Delta p \in \mathbb{Z}_{N}$, и

$$
\Lambda=\lambda_{\Delta p}=e^{2 \pi i \Delta x \Delta p}=e^{2 \pi i / N} .
$$

Соответствующие собственные векторы получаются из соотношения $\psi(x)=\widehat{T}_{x} \psi(0)$. Нормированные собственные векторы имеют вид

$$
\begin{gathered}
\psi_{\lambda_{p}}\left(x^{\prime}\right)=\left\langle x^{\prime} \mid \psi_{\lambda_{p}}\right\rangle=\frac{\lambda_{p}^{x^{\prime} / \Delta x}}{\sqrt{N}}=\frac{e^{2 \pi i x^{\prime} p}}{\sqrt{N}}, \\
\left\langle\psi_{\lambda_{p}} \mid x^{\prime \prime}\right\rangle=\left\langle x^{\prime \prime} \mid \psi_{\lambda_{p}}\right\rangle^{*}=\frac{\lambda_{p}^{-x^{\prime \prime} / \Delta x}}{\sqrt{N}}=\frac{e^{-2 \pi i x^{\prime \prime} p}}{\sqrt{N}} .
\end{gathered}
$$

Проектор на (одномерное) собственное подпространство оператора $\widehat{T}_{\Delta x}$ записывается как

$$
\begin{gathered}
\widehat{P}_{\lambda_{p}}=\left|\psi_{\lambda_{p}}\right\rangle\left\langle\psi_{\lambda_{p}}\right|, \\
\left\langle x^{\prime}\left|\widehat{P}_{\lambda_{p}}\right| x^{\prime \prime}\right\rangle=\frac{\lambda_{p}^{\left(x^{\prime}-x^{\prime \prime}\right) / \Delta x}}{N}=\frac{\lambda_{p}^{d / \Delta x}}{N}=\frac{e^{2 \pi i p d}}{N}, \quad d=x^{\prime}-x^{\prime \prime} .
\end{gathered}
$$

В данной записи $x^{\prime}$ нумерует строки матрицы, а $x^{\prime \prime}$ - столбцы.

Собственные состояния оператора минимального сдвига также являются собственными состояниями оператора импульса, поэтому их можно обозначать по-разному:

$$
\left|\psi_{\lambda_{p}}\right\rangle=\left|\psi_{p}\right\rangle=|p\rangle, \quad\langle x \mid p\rangle=\frac{e^{2 \pi i x p}}{\sqrt{N}} .
$$


2.4. Группа сдвигов. Сделаем тривиальное замечание, которое тем не менее может представлять определенный интерес. Мы строили оператор импульса так, чтобы он порождал группу симметрий относительно сдвигов координатной решетки на целое число узлов, т. е. группу, изоморфную группе (относительно сложения) остатков от деления на $N: \Delta x \cdot \mathbb{Z}_{N} \approx \mathbb{Z}_{N}$. Однако мы можем рассматривать унитарные операторы вида $\widehat{T}_{A}=e^{2 \pi i A \hat{p}}, A \in \mathbb{R}$. Такие операторы соответствуют циклическим сдвигам на произвольную величину (не обязательно кратную $\Delta x$ ). Соответствующая группа изоморфна группе $\mathbb{R} / \Xi \approx S O(1) \approx U(1)$ поворотов окружности на произвольный угол. Сложение опять понимается по модулю $\Xi, A=A+\Xi$. В случае $\Xi=\infty$ группа симметрий совпадает с группой $\mathbb{R}$ вещественных чисел относительно сложения.

Мы видим, что если гамильтониан на решетке выражается через оператор $\hat{p}$, то наличие решетки не нарушает трансляционную инвариантность по отношению к произвольным сдвигам (не обязательно на целое число узлов решетки). Однако оператор $\hat{p}$ оказывается (как мы увидим далее) нелокальным, т. е. матричные элементы $\left\langle x^{\prime}|\hat{p}| x^{\prime \prime}\right\rangle$ могут быть отличны от нуля для сколь угодно больших (в пределах решетки) значений $x^{\prime}-x^{\prime \prime}$.

Мы можем задать состояние $\left|x_{0}\right\rangle=\widehat{T}_{x_{0}}|0\rangle$ с произвольным значением $x_{0} \notin \Delta x \cdot \mathbb{Z}_{N}$, но такое состояние не будет состоянием с определенным значением координаты, поскольку оно разлагается по нескольким базисным состояниям $\{|x\rangle\}_{x \in \Delta x \cdot \mathbb{Z}_{N}}$.

\section{3. ОПЕРАТОРЫ ЦИФР И ИХ РАЗЛОЖЕНИЕ ПО СДВИГАМ}

3.1. Операторы цифр на решетке. Мы определили оператор импульса так, что фурье-гармоника импульса задает оператор $\widehat{T}_{A}=e^{2 \pi i A \hat{p}}$ сдвига по координате. Таким образом, если сделать преобразование Фурье для цифры импульса

$$
c_{n_{+} n_{-}}(r, p)=\sum_{A \in \Delta x \cdot \mathbb{Z}_{N}} \tilde{c}_{n_{+} n_{-}}(r, A) e^{2 \pi i A p},
$$

то мы получим разложение цифры импульса по координатным сдвигам:

$$
c_{n_{+} n_{-}}(r, \hat{p})=\sum_{A \in \Delta x \cdot \mathbb{Z}_{N}} \tilde{c}_{n_{+} n_{-}}(r, A) \widehat{T}_{A} .
$$

После несложных выкладок (см. приложение) получаем разложение оператора $\hat{p}_{r}$ цифры импульса частицы на решетке по операторам сдвига $\widehat{T}_{A}$ :

$$
\hat{p}_{r}=c_{n_{+} n_{-}}(r, \hat{p})=\frac{\hat{1}}{2}-\Delta p \cdot 2^{-r} \sum_{D \in \mathbb{Z}_{2^{r} / \Delta p}} \frac{\widehat{T}_{-2^{-r}(D+1 / 2)}}{1-e^{2 \pi i \Delta p \cdot 2^{-r}(D+1 / 2)}} .
$$

Аналогично оператор цифры координаты можно разложить по импульсным сдвигам $\hat{S}_{B}=e^{-2 \pi i \hat{x} B}$ :

$$
\hat{x}_{s}=c_{n_{-} n_{+}}(s, \hat{x})=\frac{\hat{1}}{2}-\Delta x \cdot 2^{-s} \sum_{D \in \mathbb{Z}_{2^{s} / \Delta x}} \frac{\hat{S}_{2^{-s}(D+1 / 2)}}{1-e^{2 \pi i \Delta x \cdot 2^{-s}(D+1 / 2)}} .
$$




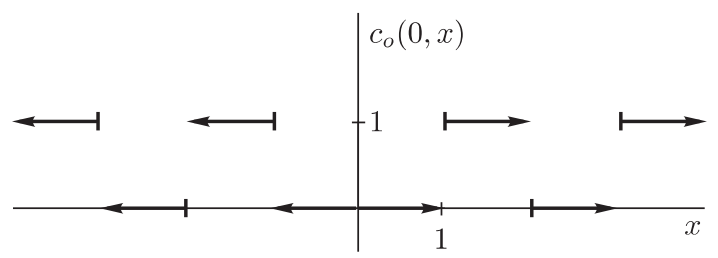

Рис. 1. График значения двоичной цифры, стоящей перед запятой (множитель при $2^{0}$ ), для обычной двоичной записи (знак задается отдельным битом) не является периодическим.

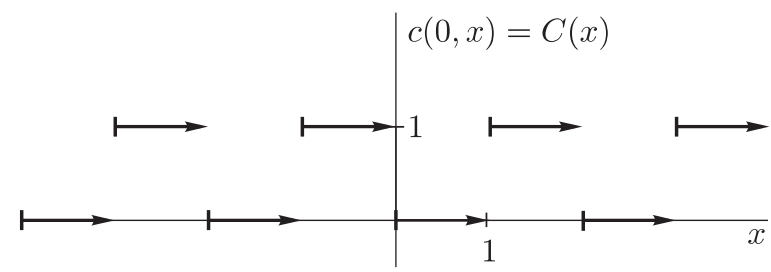

Рис. 2. График значения двоичной цифры, стоящей перед запятой (множитель при $2^{0}$ ), для двоичной записи отрицательных чисел в дополнительном коде является периодическим.

3.2. Двоичное разложение на прямой. Мы рассмотрели случай, когда координата и импульс заданы на конечной решетке. Рассмотрим переход к непрерывному пределу. Как мы увидим ниже, этот переход связан с нетривиальным обобщением суммы двоичного ряда.

Цифра двоичного разложения с номером $s \in \mathbb{Z}$ координаты $x \in \mathbb{R}$ определяется значением $x$, т. е. для каждого $s$ мы имеем функцию $c(s, x)$. Обычное двоичное разложение

$$
x= \pm \sum_{s=-\infty}^{+\infty} c_{o}(s, x) \cdot 2^{s}, \quad c_{o}(s, x) \in\{0,1\}
$$

в котором знак задается отдельным битом, не будет обобщением описанного выше формализма для решетки. Неудобство такого представления в том, что цифра $c_{o}(s, x)$ не является периодической функцией координаты $x$, что хорошо видно из графика на рис. 1. Сбой периодичности происходит за счет продолжения функции $c_{o}(s, x)$ на отрицательные значения $x$ четным образом.

Если продолжить график $c(s, x)$ на отрицательные значения $x$ периодическим образом, то мы получим другой вариант двоичного представления действительных чисел. Именно этот вариант обобщает используемое двоичное представление чисел на решетке $\Delta x \cdot \mathbb{Z}_{2^{n}}$, что легко увидеть, если применить представление кольца $\mathbb{Z}_{2^{n}}$ целыми числами от $-2^{n-1}$ до $2^{n-1}-1$.

Из графика на рис. 2 мы видим, что при изменении знака числа значение цифры меняется на дополнительное: $0 \leftrightarrow 1$. Такое представление называется двоичным представлением с записъю отрицательных чисел в дополнительном коде. Оно используется при компьютерных расчетах. 
Функцию $c(s, x)$ можно выразить через функцию одной переменной $C(x)=c(0, x)$ :

$$
c(s, x)=c\left(0,2^{-s} x\right)=C\left(2^{-s} x\right) .
$$

Удобно ввести функции $\lfloor\cdot\rfloor$ - целая часть ("пол" с округлением в меньшую сторону) и $\{\cdot\}$ - дробная часть числа,

$$
x=\lfloor x\rfloor+\{x\}, \quad\lfloor x\rfloor=\max \{n \in \mathbb{Z} \mid n \leqslant x\} .
$$

При этом

$$
C(x)=\frac{1-(-1)^{\lfloor x\rfloor}}{2}
$$

Вещественное число определяется формальным двоичным рядом:

$$
x=\sum_{s=-\infty}^{+\infty} x_{s} \cdot 2^{s}=\sum_{s=-\infty}^{+\infty} c(s, x) \cdot 2^{s} .
$$

Для положительных чисел оба представления дают одинаковые разложения. Для любого числа $x>0$ существует $s_{0} \in \mathbb{Z}$ такое, что $2^{s_{0}}<x<2^{s_{0}+1}$. Старшие цифры двоичного разложения равны нулю, $x_{S}=0$ для всех $S>s_{0}$. Ряд двоичного разложения

$$
x=\sum_{s=-\infty}^{+\infty} x_{s} \cdot 2^{s}=\sum_{s=-\infty}^{s_{0}} x_{s} \cdot 2^{s}
$$

сходится, поскольку при $s \rightarrow+\infty$ ряд обрывается, а при $s \rightarrow-\infty$ ряд мажорируется убывающей геометрической прогрессией.

Для любого числа $x<0$ существует $s_{0} \in \mathbb{Z}$ такое, что $2^{s_{0}}<|x|<2^{s_{0}+1}$. Старшие цифры двоичного разложения равны единице, $x_{S}=1$ для всех $S>s_{0}$. Ряд двоичного разложения

$$
x=\sum_{s=-\infty}^{+\infty} x_{s} \cdot 2^{s}=\sum_{s=-\infty}^{s_{0}} x_{s} \cdot 2^{s}+\sum_{s=s_{0}+1}^{+\infty} 2^{s}
$$

расходится, поскольку к сходящемуся ряду

$$
\sum_{s=-\infty}^{s_{0}} x_{s} \cdot 2^{s}=\sum_{s=-\infty}^{s_{0}}(1-c(s,|x|)) \cdot 2^{s}=2^{s_{0}+1}+x, \quad x=-|x|,
$$

добавляется сумма растущей геометрической прогрессии

$$
\sum_{s=s_{0}+1}^{+\infty} 2^{s}=2^{s_{0}+1} \sum_{s=0}^{+\infty} 2^{s} \rightarrow+\infty
$$

3.3. Перенормировка двоичных сумм. Чтобы приписать двоичному разложению отрицательного числа конечное значение $x<0$, следует провести перенормировку, которая сводится к приписыванию конечного значения сумме расходящейся геометрической прогрессии с использованием формулы $\sum_{s=0}^{+\infty} q^{s}=1 /(1-q)$ вне пре- 
делов ее применимости: для $q=2$. Перенормированную сумму будем обозначать значком суммы со штрихом:

$$
\sum_{s=0}^{+\infty} 2^{s}=\frac{1}{1-2}=-1
$$

Это же правило можно свести к виду

$$
\sum_{s=-\infty}^{+\infty} 2^{s}=0
$$

Мы могли бы рассматривать тот же ряд в 2-адическом смысле [7], но при этом мы теряем сходимость дробей с бесконечным числом ненулевых цифр после запятой и приобретаем сходимость дробей с бесконечным числом цифр до запятой. Таким образом, 2-адическая сходимость для вещественных координат оказывается неудобной, так как ее приходится применять выборочно (только для целой части отрицательных чисел). Однако мы можем рассматривать 2-адические наблюдаемые, также разлагая их по двоичным цифрам.

Чтобы применять двоичное разложение к операторам $\hat{x}$ и $\hat{p}$, полезна общая формула для отрицательных и положительных чисел. Она легко получается с помощью следующих формальных выкладок:

$$
x=2 x-x=\sum_{s=-\infty}^{+\infty} x_{s-1} \cdot 2^{s}-\sum_{s=-\infty}^{+\infty} x_{s} \cdot 2^{s}=\sum_{s=-\infty}^{+\infty}\left(x_{s-1}-x_{s}\right) \cdot 2^{s} .
$$

Легко видеть, что такой ряд сходится к $x$ вне зависимости от знака, поскольку в обоих случаях для старших степеней $x_{s-1}-x_{s}=0$. Это же правило обеспечивает обрыв ряда по отрицательным степеням, если начиная с какой-то позиции все цифры после запятой одинаковы. Таким образом, перенормированная сумма (со штрихом) имеет вид

$$
x=\sum_{s=-\infty}^{+\infty} x_{s} 2^{s}=\sum_{s=-\infty}^{+\infty}\left(x_{s-1}-x_{s}\right) \cdot 2^{s} .
$$

Это представление известно в информатике как представление чисел цифрами со знаком [8], [9]. Оно было введено с целью ускорения вычислений за счет сокращения числа переносов разрядов.

Аналогично для оператора $\hat{x}$ и его цифр мы имеем

$$
\hat{x}=\sum_{s=-\infty}^{+\infty} \hat{x}_{s} 2^{s}=\sum_{s=-\infty}^{+\infty}\left(\hat{x}_{s-1}-\hat{x}_{s}\right) \cdot 2^{s} .
$$

Цифра оператора $\hat{x}_{s}=c(s, \hat{x})$ есть проектор (самосопряженный оператор с собственными числами 0 и 1).

Мы также можем обобщить приведенный способ перенормировки на случай, когда координата задана на решетке:

$$
x^{\prime}=\sum_{s=-n_{-}}^{n_{+}-1} c(s, x) 2^{s}=\sum_{s=-n_{-}}^{n_{+}-1}(c(s-1, x)-c(s, x)) 2^{s}, \quad c\left(-n_{-}-1, x\right)=0 .
$$


Это же правило можно свести к виду

$$
\sum_{s=-n_{-}}^{n_{+}-1} 2^{s}=-2^{-n_{-}}=-\Delta x .
$$

При этом

$$
\begin{aligned}
x^{\prime} & =\sum_{s=-n_{-}}^{n_{+}-1} c(s, x) 2^{s}=2 \sum_{s=-n_{-}}^{n_{+}-2} c(s, x) 2^{s}-\underbrace{\sum_{s=-n_{-}}^{n_{+}-1} c(s, x) 2^{s}}_{=x}= \\
& =2\left(x-c\left(n_{+}-1, x\right) 2^{n_{+}-1}\right)-x=x-c\left(n_{+}-1, x\right) \underbrace{2^{n_{+}}}_{=\Xi} .
\end{aligned}
$$

Таким образом, если старшая цифра $x_{n_{+}-1}=c\left(n_{+}-1, x\right)$ равна нулю, то перенормированная координата $x^{\prime}$ положительна и совпадает с $x$, а если старшая цифра равна единице, то перенормированная координата $x^{\prime}$ отрицательна и отличается от $x$ на период решетки $\Xi, x^{\prime}=x-x_{n_{+}-1} \Xi$. Исходное выражение для $x$ соответствует представлению решетки $\Delta x \cdot \mathbb{Z}_{N}$ вещественными числами от 0 до $\Delta x \cdot(N-1)$ с шагом $\Delta x$. Перенормированная координата $x^{\prime}$ соответствует представлению той же решетки вещественными числами от $-\Delta x \cdot N / 2$ до $+\Delta x \cdot(N / 2-1)$ с шагом $\Delta x$. В информатике такая перенормировка отвечает переходу от целого положительного числа к целому числу со знаком. Для перенормированного числа старший разряд задает знак (0 соответствует знаку плюс, 1 - знаку минус).

3.4. Интегральное двоичное представление. Функция $c(s, x)$ выражается через функцию одной переменной $C(x)=c(0, x)$ по формуле (19). Мы можем рассматривать и нецелые значения $s$, что может оказаться полезным, например, при изменении масштаба единичного отрезка. Тогда $x \rightarrow 2^{a} x, c(s, x) \rightarrow c(s-a, x)$. Для положительных чисел $c(s, x)=c\left(s-\log _{2} x, 1\right), x>0$. Для отрицательных чисел $c(s, x)=1-c(s,|x|)=1-c\left(s-\log _{2}|x|, 1\right), x<0$.

По аналогии с двоичным рядом рассмотрим формальный двоичный интеграл

$$
\int_{-\infty}^{+\infty} c(s, x) 2^{s} d s
$$

Для любого $x>0$ старшие цифры равны нулю, $c(s, x)=0$ для всех $s>\log _{2} x$. Двоичный интеграл

$$
\int_{-\infty}^{+\infty} c(s, x) 2^{s} d s=\int_{-\infty}^{\log _{2} x} c(s, x) 2^{s} d s
$$

сходится, поскольку при $s \rightarrow+\infty$ подынтегральное выражение равно нулю, а при $s \rightarrow-\infty$ интеграл мажорируется сходящимся интегралом от экспоненциальной функции. Для положительных чисел легко доказать прямым вычислением (см. приложение), что двоичный интеграл, как и двоичная сумма, дает $x$ :

$$
x=\sum_{s=-\infty}^{+\infty} c(s, x) 2^{s}=\int_{-\infty}^{+\infty} c(s, x) 2^{s} d s .
$$


Для любого $x<0$ старшие цифры равны единице, $c(s, x)=1$ при всех $s \geqslant \log _{2}|x|$. Двоичный интеграл

$$
x=\int_{-\infty}^{+\infty} \underbrace{c(s, x)}_{=1-c(s,|x|)} 2^{s} d s=\int_{-\infty}^{+\infty} 2^{s} d s-\underbrace{\int_{-\infty}^{+\infty} c(s,|x|) 2^{s} d s}_{=|x|}=\int_{-\infty}^{+\infty} 2^{s} d s+x
$$

расходится, поскольку к сходящемуся интегралу добавляется расходящееся слагаемое.

Чтобы приписать двоичному интегральному представлению отрицательного числа конечное значение $x<0$, следует провести перенормировку. Аналогично двоичной сумме перенормировка соответствует применению формулы $\int_{0}^{\infty} q^{s} d s=-1 / \ln q$, верной для $0<q<1$, к случаю $q>1$ :

$$
\sum_{s=-\infty}^{+\infty} 2^{s}=\int_{-\infty}^{+\infty} 2^{s} d s=0 \Longleftrightarrow \sum_{s=0}^{+\infty} 2^{s}=\ln 2 \int_{0}^{+\infty} 2^{s} d s=-1 .
$$

Как и для двоичной суммы, можно получить общую формулу, которая сходится вне зависимости от знака величины $x$ :

$$
x=2 x-x=\int_{-\infty}^{+\infty}(c(s-1, x)-c(s, x)) \cdot 2^{s} d s .
$$

Интегральное представление является переопределенным, так как для задания числа достаточно задать его цифры с целыми номерами или цифры с номерами с шагом 1 , что соответствует выбору $2^{\Delta s}$ в качестве масштабного отрезка: для любых $\Delta s \in \mathbb{R}$

$$
x=\sum_{s=-\infty}^{+\infty} c(s+\Delta s, x) 2^{s+\Delta s}=2^{\Delta s} \sum_{s=-\infty}^{+\infty} c\left(s, x \cdot 2^{-\Delta s}\right) 2^{s} .
$$

Несмотря на переопределенность, такое представление имеет свои преимущества. В частности, интегральное представление масштабно инвариантно: оно не выделяет масштабов вида $2^{s}, s \in \mathbb{Z}$.

3.5. Операторы цифр на прямой. Функция $c(r, p)$ является периодической с периодом $2^{r+1}$, разложим ее в ряд Фурье, в результате получим разложение по операторам сдвига:

$$
\hat{p}_{r}=\sum_{A \in 2^{-r-1} \mathbb{Z}} \tilde{c}(r, A) \underbrace{e^{2 \pi i A \hat{p}}}_{=\widehat{T}_{A}}, \quad \tilde{c}(r, A)=2^{-r-1} \int_{0}^{2^{r+1}} c(r, p) e^{2 \pi i A p} d p .
$$

При этом

$$
\hat{p}_{r}=\frac{\hat{1}}{2}+\sum_{D \in \mathbb{Z}} \frac{\widehat{T}_{-2^{-r}(D+1 / 2)}}{2 \pi i(D+1 / 2)} .
$$

Такое же выражение можно получить, если перейти к пределу $n \rightarrow+\infty$ при $\Delta p \rightarrow 0$ в разложении (16) цифр импульса на решетке. Это соответствует бесконечному числу цифр до запятой у координаты и бесконечному числу цифр после запятой у импульса, т. е. решетке по координате и окружности по импульсу (если также $\Delta x \rightarrow 0$, 
то и по координате, и по импульсу получается прямая). Специально отметим, что в пределе мы получили сумму по всем целым числам включая отрицательные. До совершения предельного перехода при конечном размере решетки, замкнутой на окружность, разделение чисел на отрицательные и положительные не имело смысла.

\section{4. КОММУТАЦИОННЫЕ СООТНОШЕНИЯ}

4.1. Коммутатор цифра-цифра. Оператор цифры импульса разлагается по операторам сдвига:

$$
\hat{p}_{r}=\frac{\hat{1}}{2}-\Delta p \cdot 2^{-r} \sum_{D \in \mathbb{Z}_{2^{r} / \Delta p}} \frac{\widehat{T}_{-2^{-r}(D+1 / 2)}}{1-e^{2 \pi i \Delta p \cdot 2^{-r}(D+1 / 2)}} .
$$

Легко выводится коммутационное соотношение между произвольной функцией координаты $f(\hat{x})$ и оператором сдвига $\widehat{T}_{A}$ :

$$
\begin{aligned}
{\left[f(\hat{x}), \widehat{T}_{A}\right] \psi(x) } & =f(\hat{x}) \widehat{T}_{A} \psi(x)-\widehat{T}_{A} f(\hat{x}) \psi(x)=f(x) \psi(x+A)-f(x+A) \psi(x+A)= \\
& =(f(x)-f(x+A)) \psi(x+A)=(f(\hat{x})-f(\hat{x}+A)) \widehat{T}_{A} \psi(x) .
\end{aligned}
$$

Таким образом,

$$
\left[f(\hat{x}), \widehat{T}_{A}\right]=(f(\hat{x})-f(\hat{x}+A)) \widehat{T}_{A} .
$$

Поскольку $\hat{x}_{s}=c(s, \hat{x})$, коммутатор цифр координаты и импульса на решетке имеет вид

$$
\left[\hat{x}_{s}, \hat{p}_{r}\right]=-\Delta p \cdot 2^{-r} \sum_{D \in \mathbb{Z}_{2^{r} / \Delta p}} \frac{c(s, \hat{x})-c\left(s, \hat{x}-2^{-r}(D+1 / 2)\right)}{1-e^{2 \pi i \Delta p \cdot 2^{-r}(D+1 / 2)}} \widehat{T}_{-2^{-r}(D+1 / 2)} .
$$

На прямой коммутатор цифр координаты и импульса имеет вид

$$
\left[\hat{x}_{s}, \hat{p}_{r}\right]=\sum_{D \in \mathbb{Z}} \frac{c(s, \hat{x})-c\left(s, \hat{x}-2^{-r}(D+1 / 2)\right)}{2 \pi i(D+1 / 2)} \widehat{T}_{-2^{-r}(D+1 / 2)} .
$$

Цифра $\hat{x}_{s}$ в координатном представлении задается функцией $c(s, x)$, которая имеет период $2^{s+1}$. Величина сдвига $2^{-r-1}(2 D+1)$ является периодом функции $c(s, x)$, если

$$
\frac{2^{-r}(D+1 / 2)}{2^{s+1}}=2^{-r-s-2}(2 D+1) \in \mathbb{Z},
$$

т. е. при $-r-s-2 \geqslant 0$. Таким образом, $\left[\hat{x}_{s}, \hat{p}_{r}\right]=0$ при $s+r \leqslant-2$. В частности, дробная часть импульса коммутирует с дробной частью координаты ${ }^{1)}$, младшая цифра импульса не коммутирует только со старшей цифрой координаты, а младшая цифра координаты не коммутирует только со старшей цифрой импульса.

\footnotetext{
1) Дробные части координаты и импульса можно взять в качестве полного набора наблюдаемых для одномерного движения. Площадь такой фазовой ячейки будет составлять $h=2 \pi \hbar$ ( 1 в используемых нами единицах), это отвечает тому, что параллельный перенос по границе ячейки должен давать тождественное преобразование [10].
} 
4.2. Коммутатор координата-цифра. Поскольку $\hat{x}=\sum_{s=-n_{-}}^{n_{+}-1} 2^{s} c(s, \hat{x})$, получаем коммутатор координаты и цифры импульса на решетке:

$$
\begin{aligned}
{\left[\hat{x}, \hat{p}_{r}\right] } & =-\Delta p \cdot 2^{-r} \sum_{D \in \mathbb{Z}_{2^{r} / \Delta p}} \frac{\hat{x}-\left(\hat{x}-2^{-r}(D+1 / 2)\right)}{1-e^{2 \pi i \Delta p \cdot 2^{-r}(D+1 / 2)}} \widehat{T}_{-2^{-r}(D+1 / 2)}= \\
& =\frac{1}{2^{r}} \sum_{D \in \mathbb{Z}_{2^{r} / \Delta p}} \frac{-\Delta p \cdot 2^{-r}(D+1 / 2)}{1-e^{2 \pi i \Delta p \cdot 2^{-r}(D+1 / 2)}} \widehat{T}_{-2^{-r}(D+1 / 2)} .
\end{aligned}
$$

На прямой коммутатор координаты и цифры импульса имеет вид

$$
\left[\hat{x}, \hat{p}_{r}\right]=\frac{1}{2^{r}} \frac{1}{2 \pi i} \sum_{D \in \mathbb{Z}} \widehat{T}_{-2^{-r}(D+1 / 2)} .
$$

4.3. Коммутатор координата-импульс. Поскольку $\hat{p}=\sum_{r=-n_{+}}^{n_{-}-1} 2^{r} \hat{p}_{r}$, получаем коммутатор координаты и импульса на решетке:

$$
[\hat{x}, \hat{p}]=\sum_{r=-n_{+}}^{n_{-}-1} \sum_{D \in \mathbb{Z}_{2^{r} / \Delta p}} \frac{-\Delta p \cdot 2^{-r}(D+1 / 2)}{1-e^{2 \pi i \Delta p \cdot 2^{-r}(D+1 / 2)}} \widehat{T}_{-2^{-r}(D+1 / 2)} .
$$

На прямой получаем следующие формалъные соотношения:

$$
\begin{aligned}
{\left[\hat{x}, \hat{p}_{r}\right] } & =\frac{1}{2 \pi i \cdot 2^{r}} \sum_{D \in \mathbb{Z}} \widehat{T}_{-2^{-r}(D+1 / 2)}, \\
{[\hat{x}, \hat{p}] } & =\frac{1}{2 \pi i} \sum_{r \in \mathbb{Z}} \sum_{D \in \mathbb{Z}} \widehat{T}_{-2^{-r}(D+1 / 2)}=-i \hbar \sum_{r \in \mathbb{Z}} \sum_{D \in \mathbb{Z}} \widehat{T}_{-2^{-r}(D+1 / 2)} .
\end{aligned}
$$

Напомним, что в выбранных единицах $h=1, \hbar=1 / 2 \pi$.

4.4. Перенормировка коммутатора на прямой. Мы получили формальное разложение коммутатора в сумму операторов сдвига:

$$
[\hat{x}, \hat{p}]=-i \hbar \sum_{r \in \mathbb{Z}} \sum_{D \in \mathbb{Z}} \widehat{T}_{-2^{-r}(D+1 / 2)} .
$$

Величины сдвигов имеют вид $-2^{-r}(D+1 / 2)=-2^{-r-1}(2 D+1)$, где $r, D \in \mathbb{Z}$.

Пусть $\mathbb{A}$ - множество чисел, двоичное разложение которых содержит конечное число ненулевых множителей при отрицательных степенях двойки (конечное число значащих двоичных цифр после запятой); $\mathbb{A}$ - это группа по отношению к операции суммирования. Тогда множество величин сдвигов, по которым идет суммирование, имеет вид $\mathbb{A} \backslash\{0\}$. С учетом того, что $\widehat{T}_{0}=\hat{1}$, получаем

$$
[\hat{x}, \hat{p}]=-i \hbar \sum_{A \in \mathbb{A} \backslash\{0\}} \widehat{T}_{A}=-i \hbar\left(\sum_{A \in \mathbb{A}} \widehat{T}_{A}-\hat{1}\right)=i \hbar \hat{1}-i \hbar \sum_{A \in \mathbb{A}} \widehat{T}_{A} .
$$

Мы знаем, что для координаты и импульса частицы на прямой выполняется каноническое коммутационное соотношение $[\hat{x}, \hat{p}]=i \hbar \hat{1}$. Таким образом, мы получаем следующую перенормировку:

$$
\sum_{A \in \mathbb{A}} \widehat{T}_{A}=\sum_{A \in \mathbb{A}} e^{2 \pi i A \hat{p}}=0 .
$$

Эта перенормировка похожа на возникающее при преобразованиях Фурье формальное равенство $\int_{\mathbb{R}} e^{2 \pi i x p} d x=0$ для всех $p \neq 0$. 


\section{5. ЗАКЛЮЧЕНИЕ}

Построенное в представленной работе двоичное разложение операторов координаты и импульса может найти применение не только в области квантовых вычислений, но и при численном решении дифференциальных уравнений в частных производных на классическом компьютере. Отдельный интерес представляет естественное возникновение перенормировок. Перенормировка не только бесконечных, но и конечных (на решетке) величин позволяет численно найти перенормировку путем перехода от решетки к пределу непрерывной величины. Время и энергия также могут рассматриваться как координата и импульс [11], что позволяет применять к ним те же методы перенормировки. Перенормировки в данном контексте, вероятно, связаны с квантовой теорией измерений (о квантовой теории измерений см. [12], [13] и ссылки в этих публикациях). Представление координаты и импульса в виде двоичного разложения предполагает, что координата и импульс сами по себе не наблюдаются в эксперименте. Вместо этого непосредственно наблюдаются отдельные цифры координаты и импульса. Измерение двоичной цифры пространственной координаты соответствует прохождению/непрохождению частицы через дифракционную решетку.

\section{Вычисление цифр координаты и импульса}

ПРИЛОЖЕНИЕ А

Вычислим фурье-амплитуды цифр импульса в явном виде:

$$
\tilde{c}_{n_{+} n_{-}}(r, A)=\frac{1}{N} \sum_{p \in \Delta p \cdot \mathbb{Z}_{N}} e^{-2 \pi i p A} c_{n_{+} n_{-}}(r, p) .
$$

Очевидно, что $\tilde{c}_{n_{+} n_{-}}(r, 0)=1 / 2$. Период функции $c_{n_{+} n_{-}}(r, p)$ равен $2^{r+1}$. Количество периодов гармоники $e^{2 \pi i p A}$, которые укладываются на отрезке длины $2^{r+1}$, составляет $2^{r+1} \cdot A$. Чтобы амплитуда $\tilde{c}_{n_{+} n_{-}}(r, A)$ была отлична от нуля, это число должно быть целым. Функция $c_{n_{+} n_{-}}(r, p)$ равна нулю на первой половине периода и единице на второй. На половине периода функции $c_{n_{+} n_{-}}(r, p)$ укладывается $2^{r} \cdot A$ периодов гармоники. Чтобы амплитуда $\tilde{c}_{n_{+} n_{-}}(r, A)$ была отлична от нуля, это число не должно быть целым.

Таким образом, для ненулевых амплитуд число $2^{r} \cdot A$ должно быть полуцелым, $2^{r} \cdot A=D+1 / 2, D \in \mathbb{Z}$. Тогда

$$
\tilde{c}_{n_{+} n_{-}}(r, A)=\frac{1}{N} \cdot \frac{N \cdot \Delta p}{2^{r+1}} \sum_{p \in \Delta p \cdot \mathbb{Z}_{2^{r+1} / \Delta p}} e^{-2 \pi i p A} c_{n_{+} n_{-}}(r, p), \quad A=2^{-r}\left(D+\frac{1}{2}\right) .
$$

Поскольку $c_{n_{+} n_{-}}(r, p)$ равно нулю на первой половине периода и обращается в единицу на второй, учитывая, что $\Delta p=2^{-n_{+}}$, получаем геометрическую прогрессию:

$$
\begin{aligned}
& \tilde{c}_{n_{+} n_{-}}(r, A)=\frac{1}{2^{r+n_{+}+1}} \sum_{p \in \Delta p \cdot \mathbb{Z}_{2}{ }^{r+n_{+}+1}} e^{-2 \pi i p A} c_{n_{+} n_{-}}(r, p)= \\
& =\frac{1}{2^{r+n_{+}+1}} \sum_{p \in \Delta p \cdot \mathbb{Z}_{2}^{r+n_{+}+1}} e^{-2 \pi i p A}\left(c_{n_{+} n_{-}}(r, p)-1\right)=\frac{-1}{2^{r+n_{+}+1}} \sum_{p \in \Delta p \cdot \mathbb{Z}_{2}{ }^{r+n_{+}}} e^{-2 \pi i p A} .
\end{aligned}
$$


Сумма геометрической прогрессии вычисляется как

$$
\sum_{j=0}^{J-1} \lambda^{j}= \begin{cases}\frac{1-\lambda^{J}}{1-\lambda}, & \lambda \neq 1 \\ J, & \lambda=1\end{cases}
$$

В данном случае

$$
\begin{aligned}
& J=2^{r+n_{+}}, \quad \lambda=e^{-2 \pi i A \cdot \Delta p}=e^{-2 \pi i 2^{-r-n}+(D+1 / 2)} \neq 1, \\
& \lambda^{J}=e^{-2 \pi i(D+1 / 2)}=e^{\pi i}=-1 \text {. }
\end{aligned}
$$

Таким образом, ненулевые компоненты имеют вид

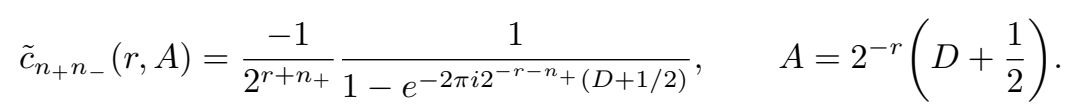

Число $D$ является целым, причем для его записи нужно $r+n_{+}$двоичных цифр, поэтому можно считать, что $D \in \mathbb{Z}_{2^{r+n_{+}}}=\mathbb{Z}_{2^{r} / \Delta p}$. Отсюда получаем разложение цифры импульса по операторам сдвига:

$$
\begin{aligned}
\hat{p}_{r} & =c_{n_{+} n_{-}}(r, \hat{p})=\sum_{A \in \Delta x \cdot \mathbb{Z}_{N}} \tilde{c}_{n_{+} n_{-}}(r, A) \underbrace{e^{2 \pi i A \hat{p}}}_{=\widehat{T}_{A}}= \\
& =\frac{\hat{1}}{2}-\Delta p \cdot 2^{-r} \sum_{D \in \mathbb{Z}_{2^{r} / \Delta p}} \frac{\widehat{T}_{-2^{-r}(D+1 / 2)}}{1-e^{2 \pi i \Delta p \cdot 2^{-r}(D+1 / 2)}} .
\end{aligned}
$$

Аналогично после замены $n_{+} \leftrightarrow n_{-}, \Delta x \leftrightarrow \Delta p, r \rightarrow s, A \rightarrow B$ получаем оператор цифры координаты через сдвиги по импульсу:

$$
\begin{aligned}
\hat{x}_{s} & =c_{n_{-} n_{+}}(s, \hat{x})=\sum_{B \in \Delta p \cdot \mathbb{Z}_{N}} \tilde{c}_{n_{-} n_{+}}(s, B) \underbrace{e^{2 \pi i B \hat{x}}}_{=\hat{S}_{-B}}= \\
& =c_{n_{-} n_{+}}(s, \hat{x})=\frac{\hat{1}}{2}-\Delta x \cdot 2^{-s} \sum_{D \in \mathbb{Z}_{2^{s} / \Delta x}} \frac{\hat{S}_{2^{-s}(D+1 / 2)}}{1-e^{2 \pi i \Delta x \cdot 2^{-s}(D+1 / 2)}} .
\end{aligned}
$$

Матричные элементы цифр импульса и координаты имеют вид

$$
\begin{aligned}
\left\langle x^{\prime}\left|\hat{p}_{r}\right| x^{\prime \prime}\right\rangle & =\frac{1}{2} \delta_{x^{\prime}, x^{\prime \prime}}-\frac{1}{2^{n_{+}+r}} \sum_{D \in \mathbb{Z}_{2^{n_{+}+r}}} \frac{\delta_{x^{\prime}, x^{\prime \prime}+2^{-r-1}(2 D+1)}}{1-e^{\pi i 2^{-n}+-r}(2 D+1)} \\
\left\langle p^{\prime}\left|\hat{x}_{s}\right| p^{\prime \prime}\right\rangle & =\frac{1}{2} \delta_{p^{\prime}, p^{\prime \prime}}-\frac{1}{2^{n_{-}+s}} \sum_{D \in \mathbb{Z}_{2^{n_{-}+s}}} \frac{\delta_{p^{\prime}, p^{\prime \prime}-2^{-s-1}(2 D+1)}}{1-e^{\pi i 2^{-n_{-}-s}(2 D+1)}}
\end{aligned}
$$

Мы можем преобразовать коэффициент следующим образом:

$$
\frac{1}{1-e^{i 2 \alpha}}=\frac{i e^{-i \alpha}}{2 \sin \alpha}=\frac{i}{2} \operatorname{ctg} \alpha+\frac{1}{2} \text {. }
$$


ПРИЛОЖЕНИЕ Б

\section{Проверка интегрального двоичного представления при $x>0$}

Докажем справедливость равенств (37) для $x>0$ прямым вычислением. Имеем

$$
c(s, x)=\left\{\begin{array}{cc}
0, & s>\log _{2} x, \\
1, & s \in\left(\log _{2} \frac{x}{2}, \log _{2} \frac{x}{1}\right], \\
0, & s \in\left(\log _{2} \frac{x}{3}, \log _{2} \frac{x}{2}\right], \\
\vdots & \vdots \\
1, & s \in\left(\log _{2} \frac{x}{2 k}, \log _{2} \frac{x}{2 k-1}\right], \\
0, & s \in\left(\log _{2} \frac{x}{2 k+1}, \log _{2} \frac{x}{2 k}\right], \\
\vdots & \vdots
\end{array} .\right.
$$

Отсюда

$$
\begin{gathered}
\int_{-\infty}^{+\infty} c(s, x) 2^{s} d s=\int_{-\infty}^{\log _{2} x} c(s, x) 2^{s} d s=\sum_{k=1}^{+\infty} \int_{\log _{2} \frac{x}{2 k}}^{\log _{2} \frac{x}{2 k-1}} e^{s \ln 2} d s=\left.\sum_{k=1}^{+\infty} \frac{1}{\ln 2} 2^{s}\right|_{\log _{2} \frac{x}{2 k}} ^{\log _{2} \frac{x}{2 k-1}}= \\
=\frac{x}{\ln 2} \sum_{k=1}^{+\infty}\left(\frac{1}{2 k-1}-\frac{1}{2 k}\right)=\frac{x}{\ln 2} \underbrace{\left(1-\frac{1}{2}+\frac{1}{3}-\frac{1}{4}+\cdots+\frac{(-1)^{n+1}}{n}+\cdots\right)}_{=\ln 2}=x .
\end{gathered}
$$

\section{Примеры}

ПРИЛОЖЕНИЕ В

Везде в этом разделе $\Delta x=1, x \in \mathbb{Z}_{N}=\{0,1, \ldots, N-1\}$, координаты и импульсы нумеруются двоичными числами, которые помечаются нижним индексом 2 , и $\Delta p=2^{-n}=1 / N$.

В.1. Случай $n=1, N=2^{1}=2$. В этом случае имеем

$$
\begin{gathered}
\hat{x}=\hat{x}_{0}=\left(\begin{array}{ll}
1 & 0 \\
0 & 0
\end{array}\right)=\frac{\hat{1}+\sigma_{z}}{2} \equiv \hat{c}, \quad \widehat{T}_{1}=\left(\begin{array}{ll}
0 & 1 \\
1 & 0
\end{array}\right) \equiv 2 \hat{h}, \\
\lambda_{0}=1=e^{2 \pi i \cdot 0}, \quad \psi_{0}=\frac{1}{\sqrt{2}}\left(\begin{array}{l}
1 \\
1
\end{array}\right) \\
\widehat{P}_{0}=\frac{1}{2}\left(\begin{array}{ll}
1 & 1 \\
1 & 1
\end{array}\right)=\frac{\hat{1}+\sigma_{x}}{2} \equiv \hat{a} \\
\lambda_{0,1_{2}}=-1=e^{2 \pi i \cdot 0,1_{2}}, \quad \psi_{0,1_{2}}=\frac{1}{\sqrt{2}}\left(\begin{array}{r}
-1 \\
1
\end{array}\right), \\
\widehat{P}_{0,1_{2}}=2 \hat{p}=\hat{p}_{-1}=\frac{1}{2}\left(\begin{array}{rr}
1 & -1 \\
-1 & 1
\end{array}\right)=\frac{\hat{1}-\sigma_{x}}{2} \equiv \hat{b}, \\
{[\hat{x}, \hat{p}]=\frac{1}{2}\left[\hat{x}_{0}, \hat{p}_{-1}\right]=\frac{1}{4}\left(\begin{array}{rr}
0 & -1 \\
1 & 0
\end{array}\right)=-\frac{i}{4} \sigma_{y} \equiv \frac{1}{2} \hat{f} .}
\end{gathered}
$$


В.2. Случай $n=2, N=2^{2}=4$. В этом случае имеем

$$
\begin{aligned}
& \hat{x}=\hat{x}_{0}+2 \hat{x}_{1}=\left(\begin{array}{cccc}
3 & 0 & 0 & 0 \\
0 & 2 & 0 & 0 \\
0 & 0 & 1 & 0 \\
0 & 0 & 0 & 0
\end{array}\right), \quad \widehat{T}_{1}=\left(\begin{array}{cccc}
0 & 0 & 0 & 1 \\
1 & 0 & 0 & 0 \\
0 & 1 & 0 & 0 \\
0 & 0 & 1 & 0
\end{array}\right), \\
& \hat{x}_{0}=\hat{1}_{2} \otimes \hat{c}=\operatorname{diag}(1,0,1,0), \quad \hat{x}_{1}=\hat{c} \otimes \hat{1}_{2}=\operatorname{diag}(1,1,0,0), \\
& \lambda_{0}=1=e^{2 \pi i \cdot 0}, \quad \psi_{0}=\frac{1}{2}\left(\begin{array}{l}
1 \\
1 \\
1 \\
1
\end{array}\right), \quad \widehat{P}_{0}=\frac{1}{4}\left(\begin{array}{cccc}
1 & 1 & 1 & 1 \\
1 & 1 & 1 & 1 \\
1 & 1 & 1 & 1 \\
1 & 1 & 1 & 1
\end{array}\right)= \\
& =\hat{a} \otimes \hat{a}, \\
& \lambda_{0,10_{2}}=-1=e^{2 \pi i \cdot 0,10_{2}}, \quad \psi_{0,10_{2}}=\frac{1}{2}\left(\begin{array}{r}
-1 \\
1 \\
-1 \\
1
\end{array}\right), \quad \widehat{P}_{0,10_{2}}=\frac{1}{4}\left(\begin{array}{rrrr}
1 & -1 & 1 & -1 \\
-1 & 1 & -1 & 1 \\
1 & -1 & 1 & -1 \\
-1 & 1 & -1 & 1
\end{array}\right)= \\
& =\hat{a} \otimes \hat{b}, \\
& \lambda_{0,01_{2}}=i=e^{2 \pi i \cdot 0,01_{2}}, \quad \psi_{0,01_{2}}=\frac{1}{2}\left(\begin{array}{r}
-i \\
-1 \\
i \\
1
\end{array}\right), \quad \widehat{P}_{0,01_{2}}=\frac{1}{4}\left(\begin{array}{rrrr}
1 & i & -1 & -i \\
-i & 1 & i & -1 \\
-1 & -i & 1 & i \\
i & -1 & -i & 1
\end{array}\right) \text {, } \\
& \lambda_{0,11_{2}}=-i=e^{2 \pi i \cdot 0,11_{2}}, \quad \psi_{0,11_{2}}=\frac{1}{2}\left(\begin{array}{r}
i \\
-1 \\
-i \\
1
\end{array}\right), \quad \widehat{P}_{0,11_{2}}=\frac{1}{4}\left(\begin{array}{rrrr}
1 & -i & -1 & i \\
i & 1 & -i & -1 \\
-1 & i & 1 & -i \\
-i & -1 & i & 1
\end{array}\right) \text {, } \\
& \hat{p}_{-1}=\widehat{P}_{0,10_{2}}+\widehat{P}_{0,11_{2}}=\frac{1}{2}\left(\begin{array}{cccc}
1 & \frac{-1-i}{2} & 0 & \frac{-1+i}{2} \\
\frac{-1+i}{2} & 1 & \frac{-1-i}{2} & 0 \\
0 & \frac{-1+i}{2} & 1 & \frac{-1-i}{2} \\
\frac{-1-i}{2} & 0 & \frac{-1+i}{2} & 1
\end{array}\right)=\frac{1}{2} \hat{1}_{2} \otimes \hat{1}_{2}-\hat{a} \otimes \hat{h}+i \hat{b} \otimes \hat{f} \\
& \hat{p}_{-2}=\widehat{P}_{0,01_{2}}+\widehat{P}_{0,11_{2}}=\frac{1}{2}\left(\begin{array}{rrrr}
1 & 0 & -1 & 0 \\
0 & 1 & 0 & -1 \\
-1 & 0 & 1 & 0 \\
0 & -1 & 0 & 1
\end{array}\right)=\frac{\hat{1}}{2}-\frac{\widehat{T}_{2}}{2}=\hat{b} \otimes \hat{1}_{2}, \\
& {\left[\hat{x}_{0}, \hat{p}_{-1}\right]=\hat{a} \otimes \hat{f}-i \hat{b} \otimes \hat{h}, \quad\left[\hat{x}_{1}, \hat{p}_{-1}\right]=\hat{f} \otimes(\hat{h}+i \hat{f}),} \\
& {\left[\hat{x}_{0}, \hat{p}_{-2}\right]=0, \quad\left[\hat{x}_{1}, \hat{p}_{-2}\right]=\hat{f} \otimes \hat{1}_{2},} \\
& \hat{p}=0,1_{2} \cdot \hat{p}_{-1}+0,01_{2} \cdot \hat{p}_{-2}=\frac{1}{8}\left(\begin{array}{cccc}
3 & -1-i & -1 & -1+i \\
-1+i & 3 & -1-i & -1 \\
-1 & -1+i & 3 & -1-i \\
-1-i & -1 & -1+i & 3
\end{array}\right) .
\end{aligned}
$$


В.3. Случай $n=3, N=2^{3}=8$. В этом случае имеем

$$
\begin{aligned}
& \hat{x}=\hat{x}_{0}+2 \hat{x}_{1}+4 \hat{x}_{2}=\left(\begin{array}{cccccccc}
7 & 0 & 0 & 0 & 0 & 0 & 0 & 0 \\
0 & 6 & 0 & 0 & 0 & 0 & 0 & 0 \\
0 & 0 & 5 & 0 & 0 & 0 & 0 & 0 \\
0 & 0 & 0 & 4 & 9 & 0 & 0 & 0 \\
0 & 0 & 0 & 0 & 3 & 0 & 0 & 0 \\
0 & 0 & 0 & 0 & 0 & 2 & 0 & 0 \\
0 & 0 & 0 & 0 & 0 & 0 & 1 & 0 \\
0 & 0 & 0 & 0 & 0 & 0 & 0 & 0
\end{array}\right), \quad \widehat{T}_{1}=\left(\begin{array}{cccccccc}
0 & 0 & 0 & 0 & 0 & 0 & 0 & 1 \\
1 & 0 & 0 & 0 & 0 & 0 & 0 & 0 \\
0 & 1 & 0 & 0 & 0 & 0 & 0 & 0 \\
0 & 0 & 1 & 0 & 0 & 0 & 0 & 0 \\
0 & 0 & 0 & 1 & 0 & 0 & 0 & 0 \\
0 & 0 & 0 & 0 & 1 & 0 & 0 & 0 \\
0 & 0 & 0 & 0 & 0 & 1 & 0 & 0 \\
0 & 0 & 0 & 0 & 0 & 0 & 1 & 0
\end{array}\right), \\
& \hat{x}_{0}=\hat{1}_{2} \otimes \hat{1}_{2} \otimes \hat{c}=\operatorname{diag}(1,0,1,0,1,0,1,0,), \\
& \hat{x}_{1}=\hat{1}_{2} \otimes \hat{c} \otimes \hat{1}_{2}=\operatorname{diag}(1,1,0,0,1,1,0,0,), \\
& \hat{x}_{2}=\hat{c} \otimes \hat{1}_{2} \otimes \hat{1}_{2}=\operatorname{diag}(1,1,1,1,0,0,0,0,), \\
& \hat{p}_{-3}=\frac{\hat{1}}{2}-\frac{\widehat{T}_{4}}{2}=\frac{1}{2}\left(\begin{array}{rrrrrrrr}
1 & 0 & 0 & 0 & -1 & 0 & 0 & 0 \\
0 & 1 & 0 & 0 & 0 & -1 & 0 & 0 \\
0 & 0 & 1 & 0 & 0 & 0 & -1 & 0 \\
0 & 0 & 0 & 1 & 0 & 0 & 0 & -1 \\
-1 & 0 & 0 & 0 & 1 & 0 & 0 & 0 \\
0 & -1 & 0 & 0 & 0 & 1 & 0 & 0 \\
0 & 0 & -1 & 0 & 0 & 0 & 1 & 0 \\
0 & 0 & 0 & -1 & 0 & 0 & 0 & 1
\end{array}\right)=\hat{b} \otimes \hat{1}_{2} \otimes \hat{1}_{2}, \\
& \hat{p}_{-2}=\frac{\hat{1}}{2}-\frac{1+i}{4} \widehat{T}_{6}-\frac{1-i}{4} \widehat{T}_{2}= \\
& =\frac{1}{2}\left(\begin{array}{cccccccc}
1 & 0 & \frac{-1-i}{2} & 0 & 0 & 0 & \frac{-1+i}{2} & 0 \\
0 & 1 & 0 & \frac{-1-i}{2} & 0 & 0 & 0 & \frac{-1+i}{2} \\
\frac{-1+i}{2} & 0 & 1 & 0 & \frac{-1-i}{2} & 0 & 0 & 0 \\
0 & \frac{-1+i}{2} & 0 & 1 & 0 & \frac{-1-i}{2} & 0 & 0 \\
0 & 0 & \frac{-1+i}{2} & 0 & 1 & 0 & \frac{-1-i}{2} & 0 \\
0 & 0 & 0 & \frac{-1+i}{2} & 0 & 1 & 0 & \frac{-1-i}{2} \\
\frac{-1-i}{2} & 0 & 0 & 0 & \frac{-1+i}{2} & 0 & 1 & 0 \\
0 & \frac{-1-i}{2} & 0 & 0 & 0 & \frac{-1+i}{2} & 0 & 1
\end{array}\right), \\
& \hat{p}_{-1}=\frac{\hat{1}}{2}-\frac{1}{4}\left(\frac{\widehat{T}_{7}}{1-e^{\pi i / 4}}+\frac{\widehat{T}_{5}}{1-e^{3 \pi i / 4}}+\frac{\widehat{T}_{3}}{1-e^{5 \pi i / 4}}+\frac{\widehat{T}_{1}}{1-e^{7 \pi i / 4}}\right)= \\
& =\frac{1}{4}\left(\begin{array}{cccccccc}
2 & -E_{1} & 0 & -E_{3} & 0 & -E_{5} & 0 & -E_{7} \\
-E_{7} & 2 & -E_{1} & 0 & -E_{3} & 0 & -E_{5} & 0 \\
0 & -E_{7} & 2 & -E_{1} & 0 & -E_{3} & 0 & -E_{5} \\
-E_{5} & 0 & -E_{7} & 2 & -E_{1} & 0 & -E_{3} & 0 \\
0 & -E_{5} & 0 & -E_{7} & 2 & -E_{1} & 0 & -E_{3} \\
-E_{3} & 0 & -E_{5} & 0 & -E_{7} & 2 & -E_{1} & 0 \\
0 & -E_{3} & 0 & -E_{5} & 0 & -E_{7} & 2 & -E_{1} \\
-E_{1} & 0 & -E_{3} & 0 & -E_{5} & 0 & -E_{7} & 2
\end{array}\right) \\
& \hat{p}=\frac{1}{8} \hat{p}_{-3}+\frac{1}{4} \hat{p}_{-2}+\frac{1}{2} \hat{p}_{-1}= \\
& =\frac{1}{16}\left(\begin{array}{cccccccc}
7 & -2 E_{1} & 4(-1-i) & -2 E_{3} & -1 & -2 E_{5} & 4(-1+i) & -2 E_{7} \\
-2 E_{7} & 7 & -2 E_{1} & 4(-1-i) & -2 E_{3} & -1 & -2 E_{5} & 4(-1+i) \\
4(-1+i) & -2 E_{7} & 7 & -2 E_{1} & 4(-1-i) & -2 E_{3} & -1 & -2 E_{5} \\
-2 E_{5} & 4(-1+i) & -2 E_{7} & 7 & -2 E_{1} & 4(-1-i) & -2 E_{3} & -1 \\
-1 & -2 E_{5} & 4(-1+i) & -2 E_{7} & 7 & -2 E_{1} & 4(-1-i) & -2 E_{3} \\
-2 E_{3} & -1 & -2 E_{5} & 4(-1+i) & -2 E_{7} & 7 & -2 E_{1} & 4(-1-i) \\
4(-1-i) & -2 E_{3} & -1 & -2 E_{5} & 4(-1+i) & -2 E_{7} & 7 & -2 E_{1} \\
-2 E_{1} & 4(-1-i) & -2 E_{3} & -1 & -2 E_{5} & 4(-1+i) & -2 E_{7} & 7
\end{array}\right),
\end{aligned}
$$

где для краткости формул введено обозначение $E_{k}=1 /\left(1-e^{k \cdot \pi i / 4}\right), k=1,3,5,7$. 
Благодарности. Автор благодарит за дискуссии участников семинара О. Г. Смолянова (мехмат МГУ, 2016), семинара "Квантовая физика и квантовая информация" (МФТИ, 2016), конференции "Новые направления в математической и теоретической физике" (МИАН, 2016). Отдельно автор благодарит И. В. Воловича, Ю. М. Белосова, Е. И. Зеленова, В. И. Манько, И. В. Маресина, Н. Н. Непойводу, Д. А. Подлесных, Д. В. Прохоренко, В. Ж. Сакбаева, М. В. Суслова, А. С. Трушечкина, Л. Е. Федичкина, С. Н Филиппова, Н. Н. Шамарова и других коллег, с которыми обсуждался материал настоящей статьи.

\section{Список литературы}

[1] L. Chen, S. Jordan, Y.-K. Liu, D. Moody, R. Peralta, R. Perlner, D. Smith-Tone, Report on Post-Quantum Cryptography, National Institute of Standards and Technology Internal Report 8105, NIST, Gaithersburg, MD, 2016.

[2] R. P. Feynman, "Simulation physics with computers", Internat. J. Theor. Phys., 21:6-7 (1982), 467-488.

[3] B. M. Boghosian, W. Taylor, "Simulating quantum mechanics on a quantum computer", Phys. D, 120:1-2 (1998), 30-42, arXiv: quant-ph/9701019.

[4] M. Bhatia, P. N. Swamy, "Elementary quantum mechanics in a space-time lattice", Internat. J. Theoret. Phys., 50:6 (2011), 1687-1698, arXiv: 1011.2544.

[5] Г. Вейль, Теория групп и квантовая механика, Наука, М., 1986.

[6] J. Schwinger, "Unitary operator bases", Proc. Nat. Acad. Sci. USA, 46:4 (1960), 570-579.

[7] В. С. Владимиров, И. В. Волович, Е. И. Зеленов, p-Адический анализ и математическая физика, Наука, М., 1994.

[8] A. Avizienis, "Signed-digit number representations for fast parallel arithmetic", IRE Transactions on Electronic Computers, EC-10:3 (1961), 389-400.

[9] C. Y. Chow, J. E. Robertson, "Logical design of a redundant binary adder", Proceedings of 4th IEEE Symposium on Computer Arithmetic (ARITH) (Santa Monica, California, October 25-27, 1978), IEEE, New York, 1978, 109-115.

[10] М. Г. Иванов, "Кривизна фазового пространства", Вестн. Сам. гос. техн. ун-та. Сер. Физ.-матем. науки, 1(30) (2013), 361-368.

[11] М. Г. Иванов, "О формулировке квантовой механики с динамическим временем", Избранные вопросы математической физики и анализа, Сборник статей. K 90-летию со дня рождения академика Василия Сергеевича Владимирова, Тр. МИАН, 285, МАИК "Наука/Интерпериодика", М., 2014, 154-165.

[12] М. Г. Иванов, Как понимать квантовую механику, РХД, М., Ижевск, 2015.

[13] М. Г. Иванов, "О единственности квантовой теории измерений для точных измерений с дискретным спектром", Тр. МФТИ, 8:1(29) (2016), 170-178.

Поступила в редакцию 10.08.2017, после доработки 26.11.2017 